\title{
Whole brain g-ratio mapping using myelin water imaging (MWI) and neurite orientation dispersion and density imaging (NODDI)
}

\author{
Woojin Jung ${ }^{1}$, Jingu Lee ${ }^{1}$, Hyeong-Geol Shin ${ }^{1}$, Yoonho Nam², Hui Zhang ${ }^{3}$, Se-Hong Oh${ }^{4}$, \\ and Jongho Lee ${ }^{1, *}$
}

\section{Author Affiliation:}

${ }^{1}$ Laboratory for Imaging Science and Technology, Department of Electrical and Computer Engineering, Seoul National University, Seoul, Korea

${ }^{2}$ Department of Radiology, Seoul St. Mary's Hospital, College of Medicine, The Catholic University of Korea, Seoul, Korea

${ }^{3}$ Department of Computer Science \& Centre for Medical Image Computing, University College London, London, United Kingdom

${ }^{4}$ Division of Biomedical Engineering, Hankuk University of Foreign Studies, Yongin, Korea

\section{Corresponding Author:}

Jongho Lee ${ }^{1}$

Department of Electrical and Computer Engineering, Seoul National University

Building 301, Room 1008, 1 Gwanak-ro, Gwanak-gu, Seoul, Korea

Tel: 82-2-880-7310

E-mail: jonghoyi@snu.ac.kr 


\begin{abstract}
MR g-ratio, which measures the ratio of the aggregate volume of axons to that of fibers in a voxel, is a potential biomarker for white matter microstructures. In this study, a new approach for acquiring an in-vivo whole human brain g-ratio map is proposed. To estimate the g-ratio, myelin volume fraction and axonal volume fraction are acquired using multi-echo gradient echo myelin water imaging (GRE-MWI) and neurite orientation dispersion and density imaging (NODDI), respectively. In order to translate myelin water fraction measured in GRE-MWI into myelin volume fraction, a new scaling procedure is proposed and validated. This scaling approach utilizes geometric measures of myelin structure and, therefore, provides robustness over previous methods. The resulting g-ratio map reveals an expected range of g-ratios $(0.71$ to 0.85 in major fiber bundles) with a small inter-subject coefficient of variance (less than $2 \%$ ). Additionally, a few fiber bundles (e.g. cortico-spinal tract and optic radiation) show different constituents of myelin volume fraction and axonal volume fraction, indicating potentials to utilize the measures for deciphering fiber tracking.
\end{abstract}

\title{
Keywords:
}

Myelin, Gradient-echo myelin water imaging (GRE-MWI), g-ratio, MRI, Diffusion, White matter microstructure 


\section{INTRODUCTION}

White matter in the brain is composed of fibers that have signal conducting axon and signal insulating myelin. The structural dimensions of these microstructures such as axonal diameter and myelin thickness are important physical parameters that determine the conduction velocity of a neuron (Goldman and Albus, 1968; Rushton, 1951; Waxman, 1980) and affect normal functions of the brain (Albert et al., 2007; Dean et al., 2016). A few studies have demonstrated that the ratio of axon diameter (excluding the thickness of myelin sheath) to fiber diameter (including the thickness of myelin sheath) is a key determinant for the conduction velocity of a fiber (Chomiak and $\mathrm{Hu}, 2009$; Goldman and Albus, 1968; Rushton, 1951; Waxman, 1980). This ratio, which is referred to as a g-ratio, may provide important scientific and clinical values (e.g. report brain plasticity from learning and capture de- and remyelination in multiple sclerosis).

However, measuring the g-ratio in-vivo is challenging because of the size of axonal fiber $(\sim \mu \mathrm{m})$ compared to MR resolution $(\sim \mathrm{mm})$. Recently, Stikov et al. have proposed an approach of measuring an area-weighted g-ratio using MRI (Stikov et al., 2011). This approach obtains the axonal and fiber volume fraction in a voxel instead of the axonal and fiber diameters of individual fibers. Then an area- (or volume-) weighted g-ratio is calculated assuming a uniform g-ratio for all fibers in the voxel.

In subsequent MR g-ratio studies, the myelin volume fraction (MVF) and axonal volume fraction (AVF) were measured using myelin-sensitive methods and diffusion-sensitive methods, respectively (Berman et al., 2017; Cercignani et al., 2017; Dean et al., 2016; Duval et al., 2017; Mohammadi et al., 2015; Stikov et al., 2015; Stikov et al., 2011). In particular, magnetization transfer (MT) imaging, such as quantitative magnetization transfer (qMT) (Cercignani et al., 2017; Stikov et al., 2015) and MT saturation (Mohammadi et al., 2015) were applied to estimate MVF, utilizing the sensitivity of the MT contrasts for myelin (Odrobina et al., 2005; Schmierer et al., 2007; Wolff and Balaban, 1989).

This approach of using an MT parameter (e.g. bounded pool fraction in qMT) for MVF requires a scaling factor that translates this parameter to MVF (Cercignani et al., 2017; Mohammadi et al., 2015; Stikov et al., 2015). To date, two approaches have been used (Table 1). The first approach estimates the scaling factor by comparing MR measurements with those of 
histology in the same ex-vivo sample (Stikov et al., 2015). The resulting scaling factor is then applied for in-vivo studies. The second approach determines the scaling factor by setting the gratio of the splenium of the corpus callosum to be 0.7 and scales the rest of the brain accordingly (Cercignani et al., 2017; Mohammadi et al., 2015). Both of these methods have critical limitations. In the first approach, a range of scaling factors (1.6 to 5.2) has been reported for different studies (Dula et al., 2010; Stikov et al., 2015; Thiessen et al., 2013) and the choice of a particular scaling factor has a significant effect on the resulting g-ratio (Campbell et al., 2017). In the second approach, no histology study exists that reports the g-ratio of the splenium in human and therefore, the choice of 0.7 as the g-ratio of splenium is arbitrary (see Discussion). Moreover, the second approach ignores g-ratio variation in the splenium among subjects. In addition, MT effects originate not only from myelin but also from other macromolecules (West et al., 2016b; Wolff and Balaban, 1989), biasing the scaling results of the MT-based approaches.

Table 1. Previous studies of the g-ratio in mammalian brains

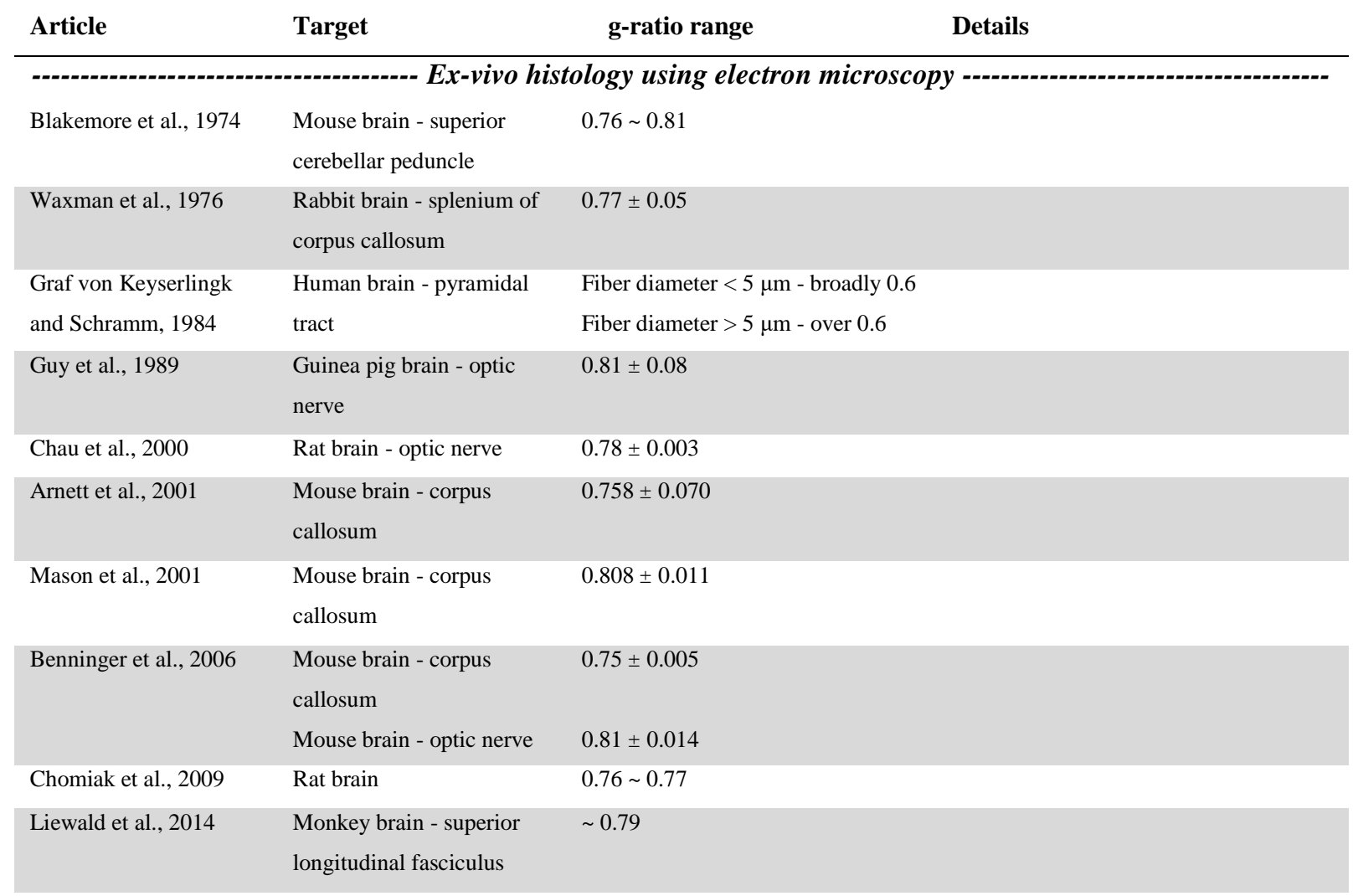




$\begin{array}{lll}\text { Stikov et al., 2015a } & \text { Macaque brain - corpus } & 0.6 \sim 0.74 \\ & \text { callosum } & \\ \text { West et al., 2016a } & \text { Mouse brain - corpus } & 0.815 \pm 0.003 \text { (mean) } \\ & \text { callosum } & 0.848 \pm 0.003 \text { (area-weighted mean) }\end{array}$

\begin{tabular}{|c|c|c|c|}
\hline \multirow[t]{2}{*}{ Stikov et al., 2015a } & Human brain & $\sim 0.7$ & $\mathrm{MT}^{1}$ and $\mathrm{NODDI}^{2}$ \\
\hline & & & Scale MT to MVF using histology \\
\hline \multirow[t]{2}{*}{ Dean et al., 2016} & Human brain & $0.71 \sim 0.9$ & mcDESPOT $^{3}$ and NODDI \\
\hline & & & Not reported \\
\hline \multirow[t]{2}{*}{ Mohammadi et al., 2016} & Human brain & $0.55 \sim 0.75$ & MT and $\mathrm{TFD}^{4}$ \\
\hline & & & Scale MT to set splenium g-ratio as 0.7 \\
\hline \multirow[t]{2}{*}{ Cercignani et al., 2017} & Human brain & $0.65 \sim 0.8$ & MT and NODDI \\
\hline & & & Scale MT to set splenium g-ratio as 0.7 \\
\hline \multirow[t]{2}{*}{ Berman et al., 2017} & Human brain - corpus & $\sim 0.69$ & $\mathrm{MTV}^{5}$ and $\mathrm{FA}^{6}$ \\
\hline & callosum & & Not required \\
\hline \multicolumn{4}{|c|}{${ }^{1}$ MT: Magnetic transfer imaging } \\
\hline \multicolumn{3}{|c|}{${ }^{3}$ mcDESPOT: Multi-component driven equilibrium single pulse observation of $\mathrm{T}_{1} / \mathrm{T}_{2}$} & ${ }^{4}$ TFD: Tensor fiber density \\
\hline \multicolumn{4}{|c|}{${ }^{5} \mathrm{MTV}$ : Lipid and macromolecular tissue volume } \\
\hline
\end{tabular}

An alternative approach for estimating MVF utilizes myelin water imaging (MWI). MWI acquires a $T_{2}\left(\right.$ or $\left.T_{2}{ }^{*}\right)$ decay curve to estimate the signal fraction of the short $\mathrm{T}_{2}\left(\right.$ or $\left.\mathrm{T}_{2}{ }^{*}\right)$ signal from myelin water and the long $\mathrm{T}_{2}$ (or $\mathrm{T}_{2} *$ ) signal from axonal and extracellular water (Du et al., 2007; Mackay et al., 1994). Then, the ratio of myelin water signal to total water signal (i.e. the sum of myelin, axonal, and extracellular water signal), which is referred to as myelin water fraction (MWF), is estimated. The method may provide a more direct measurement of myelin signal that is not complicated by other macromolecules (Laule et al., 2008; Laule et al., 2006). Recently, Dean et al., utilized MWF from mcDESPOT (Deoni et al., 2008) for g-ratio mapping without clearly detailing the conversion process from MWF to MVF (Dean et al., 2016). However, MWI signal does not include non-water components (e.g. lipid) of myelin and, therefore, a scaling process is still necessary to translate MWF into MVF.

In this study, we present an approach that scales MWF to MVF by utilizing geometry and mass-density measurements of myelin. Using this approach, an in-vivo human brain g-ratio map is generated by combining MVF from MWI and AVF from neurite orientation dispersion and 
density imaging (NODDI) (Zhang et al., 2012). Additionally, the characteristics of MVF, AVF, and g-ratio in a few major fiber bundles are analyzed. Lastly, the inter-subject coefficient of variance $(\mathrm{COV})$ is estimated to assess the reliability of the MVF, AVF, and g-ratio measurements.

\section{THEORY}

\section{Scaling myelin water fraction to myelin volume fraction}

In MWI, the signal amplitude of water compartment is determined by MR visible protons at the echo time of milliseconds. Therefore, it cannot report on structures such as myelin lipid bilayer and neurofibril. The signal amplitude of a compartment can be written as the product of the MR visible volume ratio and the volume of the compartment:

$$
A \propto \kappa \cdot V
$$

where $A$ is the signal amplitude, $\kappa$ is the MR visible volume ratio defined as the ratio of the MR visible volume to the total volume of the compartment, and $V$ is the total volume of the compartment.

Since MWI estimates the signal amplitude of the three water compartments (i.e. myelin water, axonal water and extracellular water) (Lancaster et al., 2003), MVF can be obtained using the following equation if the MR visible volume ratio of each compartment is known.

$$
M V F=V_{m y} /\left(V_{m y}+V_{a x}+V_{e x}\right)=\left(A_{m y} / \kappa_{m y}\right) /\left(A_{m y} / \kappa_{m y}+A_{a x} / \kappa_{a x}+A_{e x} / \kappa_{e x}\right)
$$

where $V_{m y}, V_{a x}$, and $V_{e x}$ are the volume of the myelin, axon, and extracellular compartments respectively, $A_{m y}, A_{a x}$, and $A_{e x}$ are the signal amplitude of the myelin water, axonal water, and extracellular water, respectively, and $\kappa_{m y}, \kappa_{a x}$, and $\kappa_{e x}$ are the MR visible volume ratios of the myelin, axon, and extracellular compartments, respectively.

In order to estimate $\kappa_{m y}$, two different methods are proposed as described as follows. The first method, which is referred to as a geometric approach, calculates $\kappa_{m y}$ using the geometric property of myelin. The second method calculates the value using the mass and density of myelin 
and is referred to as a mass-density approach.

The geometric approach utilizes the lamellar structure of myelin sheath, which is composed of four types of layers (i.e. lipid bilayer, major dense line, lipid bilayer, and intraperiod line) (Peters, 1960). Assuming that only protons in the major dense line and intraperiod line contribute to the myelin water signal and that the contribution from other molecules (e.g. proteins) can be ignored, we can estimate the MR visible volume ratio of the myelin compartment using the thicknesses of the layers, which are reported in X-ray diffraction studies of mammalian CNS nerves (Finean, 1962; Morell and Quarles, 1999). Considering the length $(l)$ of myelinated axon with a particular number of lamellae (n) (Fig. 1), myelin lipid volume (MLV) and myelin water volume (MWV) are calculated as the sum of the alternating volumes of the concentric cylinders (i.e. yellow areas $\times l$ for MLV and blue areas $\times l$ for MWV in Fig. 1a):

$$
M L V=\sum_{i=0}^{2 n} \pi\left[\left\{\left(w_{\text {lip }}+w_{\text {water }}\right) \times i+w_{\text {lip }}+r_{\text {ax }}\right\}^{2}-\left\{\left(w_{\text {lip }}+w_{\text {water }}\right) \times i+r_{a x}\right\}^{2}\right] \times l
$$

$$
M W V=\sum_{i=1}^{2 n} \pi\left[\left\{\left(w_{\text {lip }}+w_{\text {water }}\right) \times i+r_{a x}\right\}^{2}-\left\{\left(w_{\text {lip }}+w_{\text {water }}\right) \times i-w_{\text {water }}+r_{a x}\right\}^{2}\right] \times l
$$

where $w_{\text {lip }}$ and $w_{\text {water }}$ represent the thickness of the lipid layer and water layer, $r_{a x}$ is the inner radius of the axon, and $i=0$ indicates a myelinated axon with a single lipid bilayer (Fig. 1a). Then, the MR visible volume ratio of the myelin compartment $\left(\kappa_{m y}\right)$ can be written as

$$
\kappa_{m y}=M W V /(M W V+M L V)=w_{\text {water }} /\left\{(1+1 / 2 n) w_{\text {lip }}+w_{\text {water }}\right\} .
$$

Thus, once the number of the lamella is known, the MR visible volume ratio of the myelin compartment can be obtained. Note that $\kappa_{m y}$ is not dependent on $r_{a x}$ because it cancels out when Eq. 5 is expanded. Since this approach is based on the geometric parameters of myelin, it is named as the geometric approach.

Another approach for calculating $\kappa_{m y}$ uses mass-density measurements (Dula et al., 
2010; Laule et al., 2004; West et al., 2016b) and is referred to as the mass-density approach. Since volume is defined as mass divided by (buoyant) density, MWV and MLV can be expressed as follows:

$$
\begin{gathered}
M W V=m_{\text {myelin water }} / \rho_{\text {myelin water }} \\
M L V=m_{\text {myelin lip }} / \rho_{\text {myelin lip }}
\end{gathered}
$$

where $m_{\text {myelin water }}$ and $m_{\text {myelin lip }}$ represent the mass (fraction) of myelin water layer and myelin lipid layer, respectively and $\rho_{\text {myelin water }}$ and $\rho_{\text {myelin lip }}$ are the density of myelin water layer and myelin lipid layer respectively. Then, $\kappa_{m y}$ can be estimated from literature values of each parameter (Laule et al., 2004).

The same approach can be applied to estimate the MR visible volume ratios of the axon and extracellular compartments. Assuming that the two compartments have the same ratio, we can combine the two compartments and divide the volume into water volume (axonal \& extracellular water volume; AWV) and non-water volume (axonal \& extracellular non-water volume; ANWV). Then, each volume can be written as

$$
\begin{gathered}
A W V=m_{\text {axon } \& \text { ext water }} / \rho_{\text {axon } \& \text { ext water }} \\
A N W V=m_{\text {axon \& ext non-water }} / \rho_{\text {axon } \& \text { ext non-water }}
\end{gathered}
$$

where $m_{\text {axon } \& \text { ext water }}$ and $m_{\text {axon } \& \text { ext non-water }}$ represent the mass (fraction) of axonalextracellular water and axonal-extracellular non-water, respectively, and $\rho_{\text {axon } \& \text { ext water }}$ and $\rho_{\text {axon } \& \text { ext non-water }}$ represent the density of axonal-extracellular water and axonalextracellular non-water, respectively. Then, similar to Eq. 5, $\kappa_{a x}$ and $\kappa_{e x}$ can be obtained using

$$
\kappa_{a x}=\kappa_{e x}=A W V /(A W V+A N W V) .
$$

These MR visible volume ratios of the three compartments are combined with the signal amplitude measurements of MWI to estimate MVF using Eq. 2.

\section{MATERIALS AND METHODS}

Parameters of the scaling factors 
The parameters of the scaling factors used literature values of mammalian CNS. In the geometric approach, the thicknesses of lipid bilayer $\left(=w_{\text {lip }}\right)$ and water layer $\left(=w_{\text {water }}\right)$ were set

to be $51 \AA$ and $29 \AA$, respectively (Finean, 1962; Morell and Quarles, 1999). In the mass-density approach, the mass fractions and densities of myelin water and lipid layers were as follows: $m_{\text {myelin water }}=0.082 \mathrm{~g}$ per $1 \mathrm{~g}$ of white matter, $m_{\text {myelin lip }}=0.14 \mathrm{~g}$ per $1 \mathrm{~g}$ of white matter, $\rho_{\text {myelin water }}=1 \mathrm{~g} / \mathrm{ml}$, and $\rho_{\text {myelin lip }}=1.08 \mathrm{~g} / \mathrm{ml}$ (Laule et al., 2004). For the calculation of $\kappa_{a x}$ and $\kappa_{e x}$, the following parameters were used for mass fractions and densities of water and non-water components in axonal and extracellular space: $m_{\text {axon } \& \text { ext water }}=0.638 \mathrm{~g}$ per $1 \mathrm{~g}$ of white matter, $m_{\text {axon } \& \text { ext non-water }}=0.14 \mathrm{~g}$ per $1 \mathrm{~g}$ of white matter, $\rho_{\text {axon }}$ \& ext water $=1$ $\mathrm{g} / \mathrm{ml}$, and $\rho_{\text {axon } \& \text { ext non-water }}=1.33 \mathrm{~g} / \mathrm{ml}$ (Laule et al., 2004).

\section{Validation of the scaling factors using existing histological data}

To validate the scaling approaches, an existing histo-imaging dataset was utilized (West et al., 2016b). The dataset contained histologically measured MVF and MR measured MWF. Four different ROIs were chosen (genu, mid-body, splenium of corpus callosum, and anterior commissure) from four different types of mouse groups (two hypomyelinated mice ( $\mathrm{n}=3$ each), one hypermyelinated $(n=3)$, and control mouse groups $(n=6)$ ), yielding a wide range of MVF. To demonstrate the validity of proposed scaling methods, the scaling factors from the geometric approach and the mass-density approach were applied to translate the MRI MWF in the dataset into MRI MVF. Then, a linear regression was applied to MRI MVF and histological MVF, and $\mathrm{R}^{2}$ was calculated to demonstrate the goodness of fit.

\section{MRI data acquisition}

Five healthy subjects were scanned at a 3 Tesla MRI system (Tim Trio, SIEMENS, Erlangen, Germany) using a 32-channel phased-array head coil. The study was performed under the approval of the internal review board. Multi-echo GRE images and multi-shell diffusion images were acquired for MWI and NODDI, respectively. 
The scan parameters for the multi-echo GRE were as follows: multi-slice acquisition, TR $=1400 \mathrm{~ms}, \mathrm{TE}=2.45 \mathrm{~ms}$ to $39.5 \mathrm{~ms}$ with $2.2 \mathrm{~ms}$ echo spacing, number of echoes $=18$, flip angle $=83^{\circ}$, bandwidth $=500 \mathrm{~Hz} /$ pixel, FOV $=256 \times 208 \mathrm{~mm}^{2}$, voxel size $=2 \times 2 \mathrm{~mm}^{2}$, slice thickness

$=2 \mathrm{~mm}$, number of slices $=48$, concatenation $=2$, bipolar readout gradient, and scan time $=5 \mathrm{~min}$. Both respiratory and cardiac noise were reduced using navigation echoes $\left(17^{\text {th }}\right.$ and $18^{\text {th }}$ echoes) and flow saturation RF pulses (Nam et al., 2015a). To compensate for the eddy current effects from the bipolar readout gradient, the scan was repeated using the opposite readout direction (Lu et al., 2008).

For NODDI, three-shell diffusion weighted imaging data $\left(b=300 \mathrm{~s} / \mathrm{mm}^{2}\right.$ with 8 directions; $\mathrm{b}=700 \mathrm{~s} / \mathrm{mm}^{2}$ with 32 directions; $\mathrm{b}=2000 \mathrm{~s} / \mathrm{mm}^{2}$ with 64 directions; $\mathrm{b}=0 \mathrm{~s} / \mathrm{mm}^{2}$ with 13 averages) was acquired using a single shot spin-echo echo-planar-imaging (SE-EPI) sequence with TR = $4000 \mathrm{~ms}, \mathrm{TE}=95 \mathrm{~ms}, \mathrm{FOV}=192 \times 192 \mathrm{~mm}^{2}$, voxel size $=2 \times 2 \mathrm{~mm}^{2}$, slice thickness $=2 \mathrm{~mm}$, multi-band factor $=2$, partial Fourier $=6 / 8$, GRAPPA factor $=2$, and scan time $=9.75 \mathrm{~min}$. To compensate for EPI geometric distortion, additional $b=0 \mathrm{~s} / \mathrm{mm}^{2}$ image was acquired with the opposite phase encoding direction. The geometric distortion was corrected using TOPUP and EDDY (FSL, FMRIB, Oxford, UK) (Andersson et al., 2003).

\section{MRI data processing}

The multi-echo GRE data were processed as follows: First, respiration induced phase errors were reduced using the navigation echoes (Nam et al., 2015a). Gibbs' ringing was attenuated using a Tukey window (filter size $=1 / 3$ ) applied in k-space. Then, multi-channel images were combined by the root sum-of-squared for magnitude and by the complex sum for phase after phase offset correction (Hammond et al., 2008). Eddy current induced artifacts from the bipolar readout were compensated by combining the two GRE images of the opposite readout directions (Buonocore and Gao, 1997).

After generating the combined GRE images, $A_{m y}, A_{a x}$, and $A_{e x}$ were estimated by fitting a complex three pool model using a nonlinear-least square curve fitting algorithm (lsqnonlin function in MATLAB, TolX = 1e-5, TolFun = 1e-5) with the fitting parameters described in Nam 
et al. (Nam et al., 2015b). Finally, the resulting signal amplitudes $\left(A_{m y}, A_{a x}\right.$, and $\left.A_{e x}\right)$ were used to calculate MVF using Eq. 2.

For AVF, the diffusion images were processed using AMICO (Daducci et al., 2015). In NODDI, the volume of each voxel was divided into isotropic $\left(V_{i s o}\right)$ and anisotropic volume fraction $\left(1-V_{i s o}\right)$. Subsequently, the anisotropic volume fraction was divided into anisotropically restricted volume fraction $\left(\left(1-V_{i s o}\right) V_{i c}\right)$, originating from axonal space, and anisotropically hindered volume fraction $\left(\left(1-V_{i s o}\right)\left(1-V_{i c}\right)\right)$, originating from extracellular space (Zhang et al., 2012). When calculating these volume fractions, a method that corrects for $\mathrm{T}_{2}$ difference between isotropic compartment (i.e. CSF) and anisotropic compartment has been applied using $\mathrm{T}_{2}$ values of $2000 \mathrm{~ms}$ and $90 \mathrm{~ms}$, respectively (Bouyagoub et al., 2016).

Since the myelin signal was not included in NODDI acquisition due to the long TE of diffusion weighted imaging, the estimated volume fraction was scaled by $(1-M V F)$ (Stikov et al., 2015), which was obtained in MWI. Then, the anisotropically restricted volume fraction was transformed into AVF using the following equation:

$$
A V F=(1-M V F)\left(1-V_{i s o}\right) V_{i c}
$$

This procedure of combining the anisotropically restricted volume fraction and myelin volume fraction requires an accurate registration between the diffusion and GRE images. The registration was performed between the $5^{\text {th }}$ echo GRE image and the $b=0 \mathrm{~s} / \mathrm{mm}^{2}$ diffusion image using BSpline in Advanced Normalization Tools (ANTs) (Avants et al., 2009) after brain extraction (Jenkinson et al., 2005). The resulting transformation matrix was applied to the MVF map.

Finally, an aggregate g-ratio was calculated from MVF and AVF values using the following equation (Stikov et al., 2015):

$$
g=\sqrt{1 /(1+M V F / A V F)}
$$

This aggregate g-ratio was calculated in each voxel, generating an in-vivo g-ratio map of the brain.

Additionally, the diffusion weighted images (all $b$ values) were processed to identify major fiber bundles. DTI reconstruction was performed using FSL's DTIFIT (Jenkinson et al., 2012). 


\section{MRI data analysis}

A white matter mask was created for brain voxels with MVF ranging from 0.01 to 0.50 and AVF higher than 0.2. The resulting mask was smoothed by a Gaussian filter (standard deviation $=2$ voxels) and then thresholded at 0.6 , generating the final white matter mask. Six regions of interest (ROIs), cingulum bundles (CG), cortico-spinal tract (CST), genu (GN), optic radiation (OR), superior longitudinal fasciculus (SLF), and splenium (SPL) were manually chosen based on the color-coded DTI FA map in each subject (Supplementary Fig. S1). The mean MVF, AVF and g-ratio values of each ROI were computed in each subject.

To demonstrate the reliability of the g-ratio map, the inter-subject COV was calculated as follows:

Inter-subject COV of an ROI = (standard deviation of the ROI across subjects) $)$

(average of the ROI across subjects).

(Eq. 13)

Additionally, a scatter plot of MVF and AVF for each subject was generated to demonstrate different constitution of the volume fractions in the fiber tracts.

\section{RESULTS}

\section{Scaling myelin water fraction to myelin volume fraction}

Fig. $1 \mathrm{~b}$ shows the MR visible volume ratio of the myelin compartment $\left(\kappa_{m y}\right)$ as a function of the number of lamellae using the geometric approach. The plot reveals that $\kappa_{m y}$ ranges from 0.34 to 0.36 for a myelinated fiber that has more than five lamellae. When the average number of lamellae $(=15)$ in mammalian CNS nerves (Mierzwa et al., 2010) is used, $\kappa_{m y}$ is approximately 0.36. In the mass-density approach, $\kappa_{m y}$ is calculated to be 0.39 (Eqs. 6 and 7), which is close to $\kappa_{m y}$ from the geometric approach. For $\kappa_{a x}$ and $\kappa_{e x}$, the result of the mass-density approach (Eqs. 8 to 10$)$ was 0.86 . 


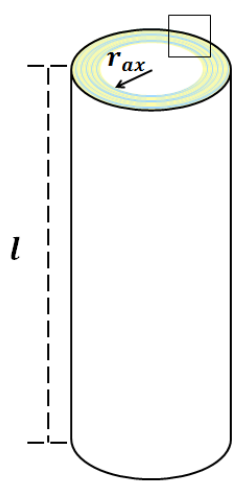

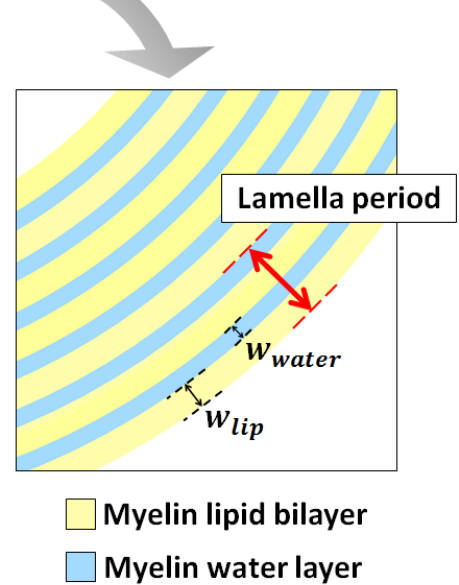

(a)

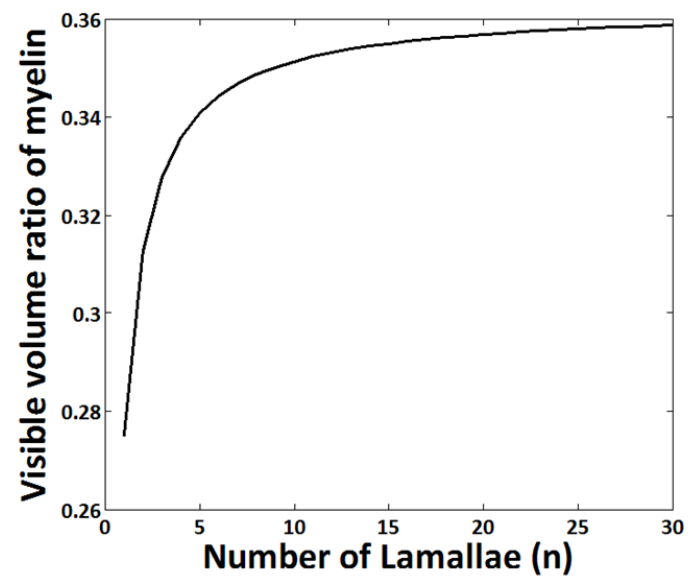

(b)

Figure 1. (a) Schematic diagram of a myelinated axon. One lamella consists of four layers alternating between water and lipid (red arrow). (b) MR visible volume ratio of the myelin compartment $\left(\kappa_{m y}\right)$ as a function of the number of lamellae (solid black line) in the geometric approach. 


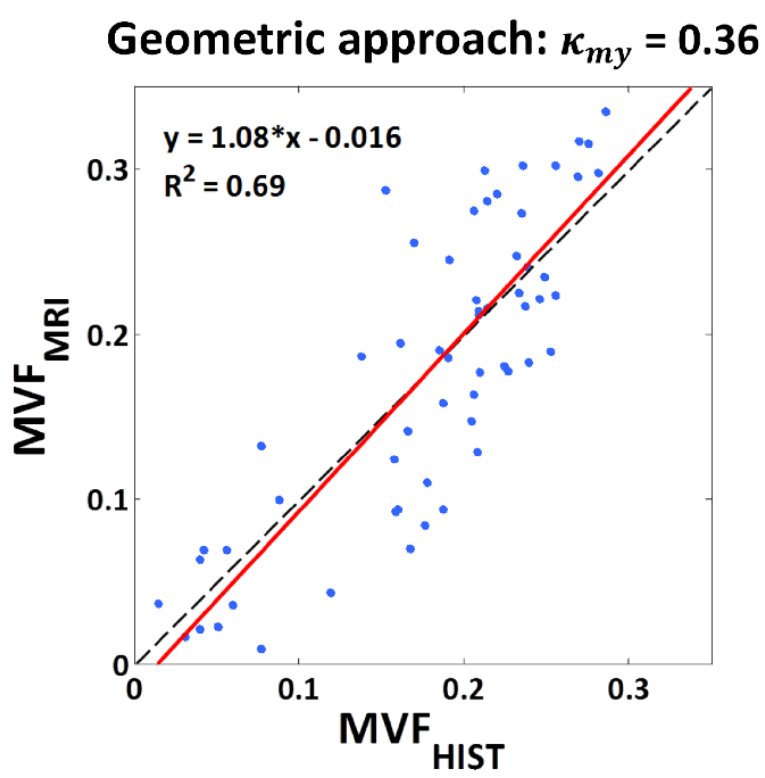

(a)

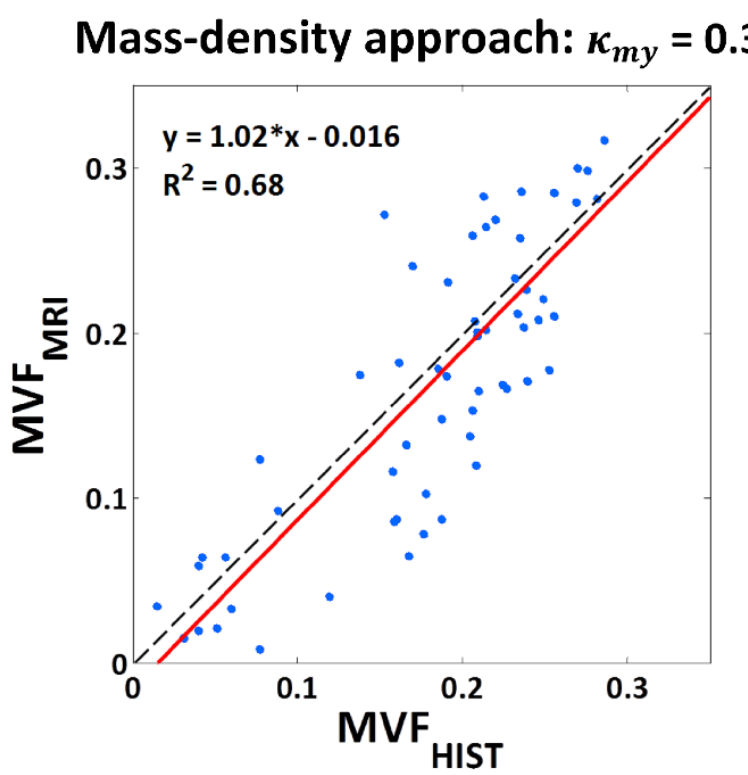

(b)

Figure 2. Validation of the scaling approaches using existing histo-imaging data (West et al., 2016b). Histologically measured MVF and MRI estimated MVF scaled from MWI using the two proposed scaling methods are plotted (left: scaling using the geometric approach, right: scaling using the mass-density approach). Solid red lines, which represent linear regression lines, are close to the line of unity (dashed black line), suggesting good correspondences between the two measurements.

When these MR visible volume ratios are applied to the existing histo-imaging dataset (West et al., 2016b), the results show good correspondences between histological MVF and MRI MVF as plotted in Fig. 2. Each blue circle represents an ROI measurement with an $x$ value as histological MVF and a y value as MRI MVF translated from MWF. The solid red line is the result of a linear regression whereas the dashed black line is the line of unity. The linear regression lines are close to the line of unity with high $\mathrm{R}^{2}$ (0.69 for the geometric approach in Fig. 2a; 0.68 for the mass-density approach in Fig. 2b), confirming that the proposed scaling approaches are valid. Hence, the MR visible volume ratios of the three compartments, $\kappa_{m y}=0.36$ and $\kappa_{a x}=\kappa_{e x}=$ 0.86 , are used hereafter. When these volume ratios are applied in Eq. 2, MWF is translated into 
MVF as shown in Fig. 3.

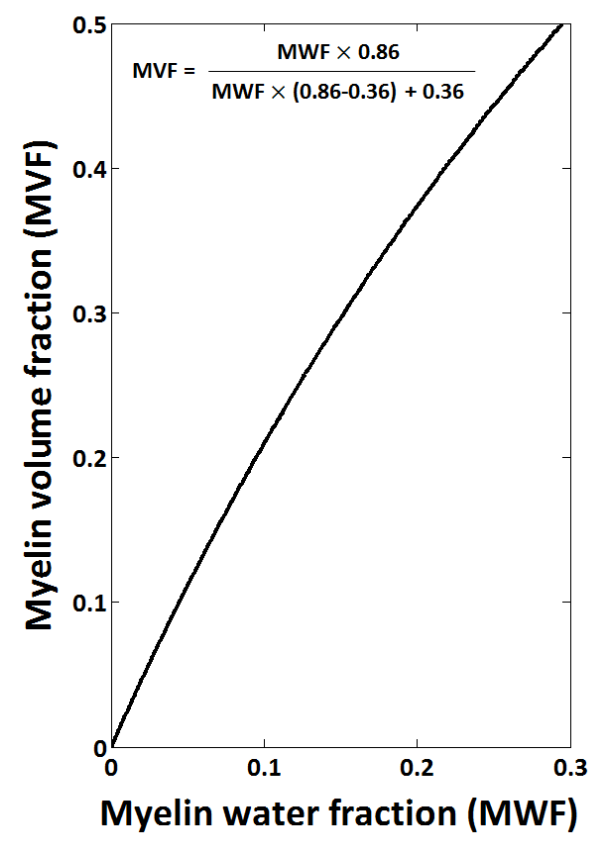

Figure 3. Plots between myelin water fraction and myelin volume fraction using the geometric approach.

In-vivo human brain g-ratio mapping 


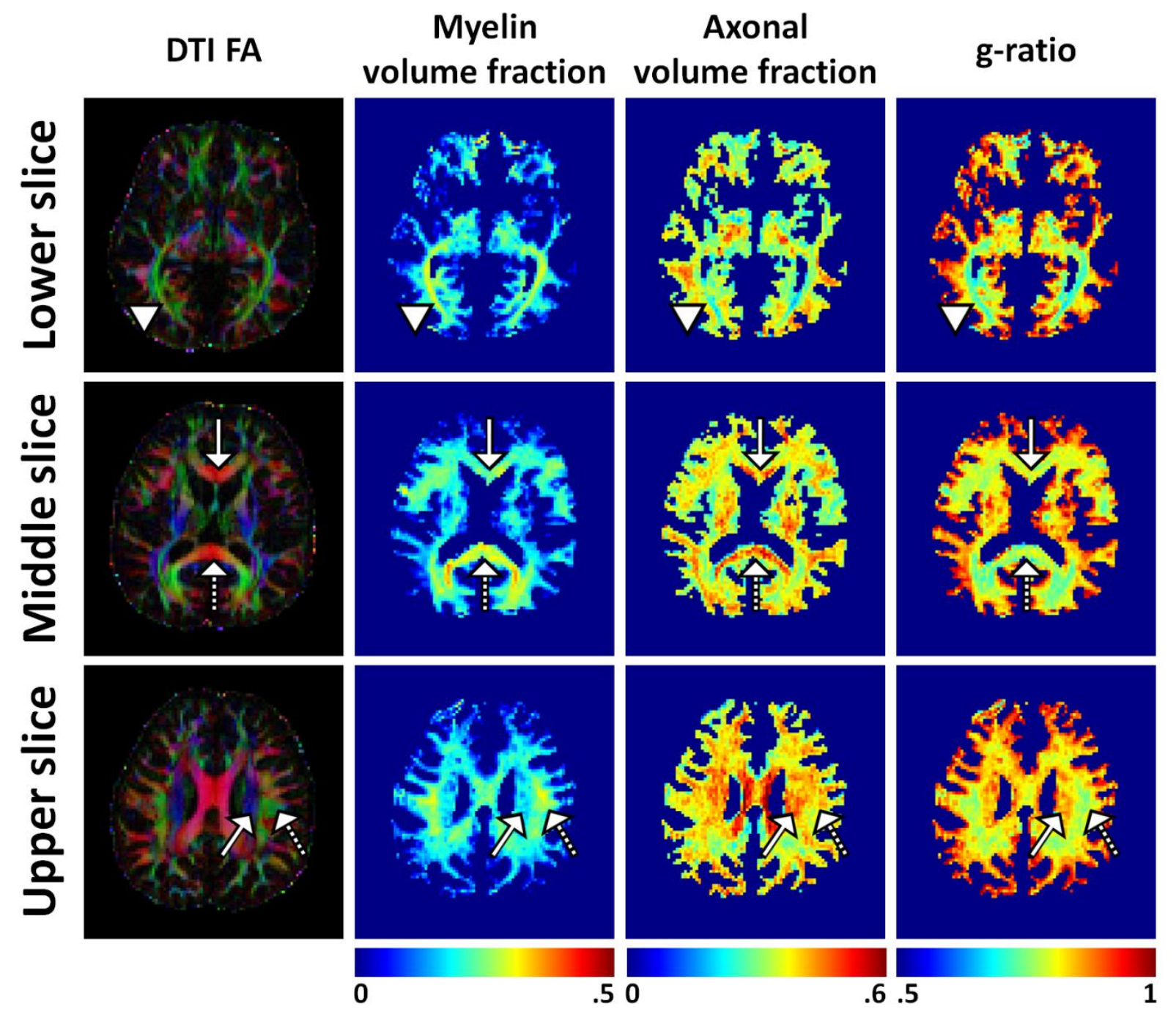

Figure 4. Color-coded DTI FA (a), myelin volume fraction (MVF) (b), axonal volume fraction $(A V F)(c)$, and g-ratio (d) maps in white matter are displayed for three different slices. The triangle in the lower slice indicates optic radiation (OR). The solid line arrow and dashed line arrow in the middle slice indicate genu (GN) and splenium (SPL), respectively. In the upper slice, the solid line arrow and dashed line arrow indicate cortico-spinal tract (CST) and superior longitudinal fasciculus (SLF), respectively.

The color-coded DTI FA, MVF, AVF, and g-ratio maps of a representative subject are shown in Fig. 4. Three slices that contains the major fiber bundles are displayed. In the lower slice, 
the triangle in Fig. 4 indicates OR, which shows high MVF $(0.28 \pm 0.01$; all values hereafter are group-averaged results) and low $\operatorname{AVF}(0.29 \pm 0.01)$, resulting in a low g-ratio $(0.71 \pm 0.01)$. In the middle slice, the solid line arrow shows $\mathrm{GN}(\mathrm{MVF}=0.26 \pm 0.02, \mathrm{AVF}=0.43 \pm 0.02$, and g-ratio $=0.79 \pm 0.01)$ and the dashed line arrow indicates $\mathrm{SPL}(\mathrm{MVF}=0.30 \pm 0.01, \mathrm{AVF}=0.44 \pm 0.02$, and g-ratio $0.77 \pm 0.01$ ). In the upper slice, the arrow with dashed line indicates SLF (MVF $=0.29$ $\pm 0.01, \mathrm{AVF}=0.38 \pm 0.02$, and g-ratio $=0.76 \pm 0.01)$ and the arrow with solid line indicates CST $(\mathrm{MVF}=0.16 \pm 0.01, \mathrm{AVF}=0.43 \pm 0.02$, and g-ratio $=0.85 \pm 0.003)$. The g-ratio values are markedly higher in CST than in SLF (CST $=0.85 \pm 0.003$ vs. SLF $=0.75 \pm 0.01$ ), which is explained by the lower MVFs in CST than in SLF (CST $=0.16 \pm 0.01$ vs. SLF $=0.29 \pm 0.01$ ) while the AVFs are similar $(\mathrm{CST}=0.43 \pm 0.02$ vs. $\mathrm{SLF}=0.38 \pm 0.02)$.

\section{Myelin volume fraction, axonal volume fraction and g-ratio}

Fig. 5 shows the average values of MVF, AVF, and g-ratio in each ROI for each subject. Each bar represents the average value of an ROI from each subject with the standard deviation. The subject-averaged MVF was $0.26 \pm 0.05$ (from 0.16 to 0.30 ), that of AVF was $0.39 \pm 0.06$ (from 0.29 to 0.44 ), and that of g-ratio was $0.78 \pm 0.05$ (from 0.71 to 0.85 ). Overall, the inter-subject variation of an ROI was relatively small in all parameters. The inter-subject COV of the MVF, AVF, and g-ratio are reported in Table 2. Most of the COVs except MVF in GN are less than 6\%, suggesting the measurements are consistent across the subjects (Table 2). 

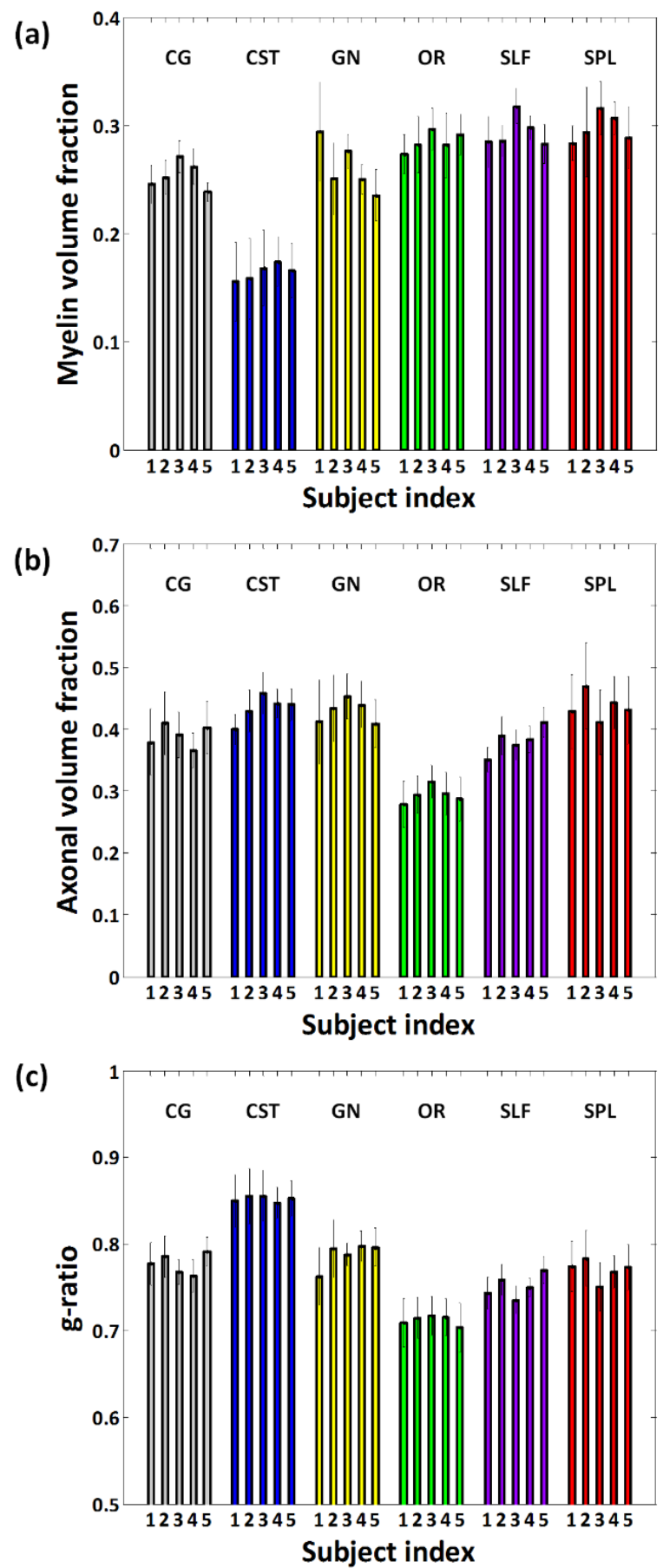

Figure 5. Mean myelin volume fraction (a), axonal volume fraction (b), and aggregate g-ratio (c) with the standard deviation of the ROIs for each subject are plotted (CG: cingulum, CST: corticospinal tract, GN: genu, OR: optic-radiation, SLF: superior longitudinal fasciculus, and SPL: splenium). 
Table 2. The inter-subject coefficient of variance (COV) of the MVF, AVF, and g-ratio in each ROI across the subjects (CG: cingulum, CST: cortico-spinal tract, GN: genu, OR: optic-radiation, SLF: superior longitudinal fasciculus, and SPL: splenium)

\begin{tabular}{ccccccc} 
& \multicolumn{7}{c}{ Inter-subject coefficient of variance (\%) } \\
\cline { 2 - 7 } & CG & CST & GN & OR & SLF & SPL \\
\hline MVF & 5.1 & 4.6 & 9.0 & 3.2 & 5.0 & 4.5 \\
AVF & 4.5 & 5.0 & 4.4 & 4.5 & 5.7 & 4.9 \\
g-ratio & 1.5 & 0.4 & 1.9 & 0.8 & 1.8 & 1.5
\end{tabular}

The scatter plot of ROI-averaged MVF and AVF for each subject is shown in Fig. 6. The plot reveals isolated clusters for CST (low MVF and high AVF) and OR (high MVF and low AVF) while the others ROIs are relatively close to each other (high MVF and high AVF). Still, it may be possible to further distinguish SLF (high MVF and mid AVF), CG (mid MVF and mid AVF), and SPL (high MVF and high AVF), if GN, which may be affected by the field inhomogeneity artifacts, is ignored.

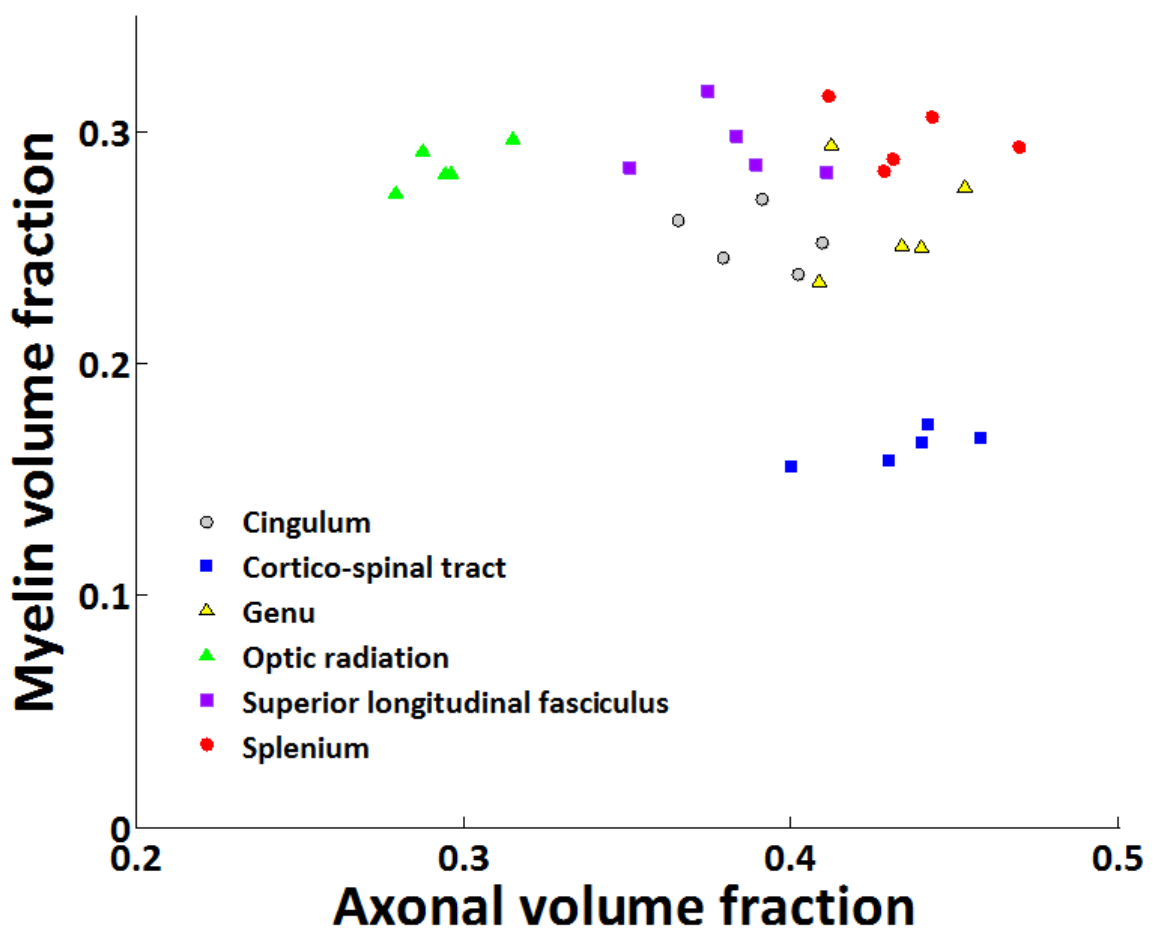


Figure 6. Scatter plot of ROI-averaged myelin volume fraction and axonal volume fraction in each subject for the six ROIs. Cortico-spinal tract and optic radiation form distinct clusters.

\section{DISCUSSION}

In this study, we developed an approach to generate an in-vivo whole human brain g-ratio map by combining MWI and NODDI. The scaling methods that translated MWF into MVF demonstrated a good correspondence with the histological results. The resulting g-ratio map showed consistency across the subjects with small inter-subject COVs. Additionally, we demonstrated that AVF and MVF of a few major fiber bundles have a distinct constituent, which may be helpful in resolving crossing fibers in fiber tracking. The proposed method takes less than 20 min. of scan time and can be applied in a clinical setting.

In our results, the g-ratio varied from $0.71 \pm 0.01(\mathrm{OR})$ to $0.85 \pm 0.01(\mathrm{CST})$. The high gratio of CST is because of the low MVF $(0.16 \pm 0.01)$ and the high AVF $(0.43 \pm 0.02)$, which is partly supported by the histological finding of large axons in CST (Graf von Keyserlingk and Schramm, 1984; Yagishita et al., 1994). The low g-ratio of OR is due to a high MVF $(0.28 \pm 0.01)$ and a low AVF $(0.29 \pm 0.01)$. This is explained in part by the thick myelin sheath in OR (Kitajima et al., 1996). Unfortunately, there is no histological reference of human g-ratio except Graf von Keyserlingk and Schramm's study, which only reports rough g-ratios of pyramidal tract (Table 1). Further investigation of fiber tract-wise g-ratio in the human brain is necessary.

One of the interesting results of our study is that the two quantities, MVF and AVF, used for the g-ratio calculation provide important information of the white matter microstructure that can be used to distinguish different fiber bundles as demonstrated in Fig. 6. This information may be helpful in disentangling crossing fibers using an approach similar to the one proposed by De Santis et al., (De Santis et al., 2016). Hence, reporting and utilizing all three parameters will be valuable for future research.

\section{Robustness of the scaling factors}


In this study, we demonstrated that the scaling parameters of both geometric and massdensity approaches were similar (0.36 and 0.39, respectively) despite differences in the methods. Additionally, we showed that these parameters were consistent with the results of the histoimaging data (West et al., 2016b), suggesting robustness of the scaling factors. Still, our method assumed consistent scaling parameters across fibers, regions, and species. In particular, the geometric approach considered the thicknesses of myelin water layer $\left(w_{\text {water }}\right)$ and lipid layer $\left(w_{\text {lip }}\right)$ or the ratio of the two thickness $\left(w_{\text {lip }} / w_{\text {water }}\right)$ to be the same. To our knowledge, two measurements of the thicknesses from two different species (rat optic nerve (Finean, 1962) and rabbit optic nerve (Kirschner and Caspar, 1972); both used fresh tissues) exist in literature and they reported similar results (the thickness ratio was 1.73 in rat and 1.55 in rabbit). When a computer simulation was performed to estimate the effects of this variation in MVF and g-ratio, the results showed very small differences (see Supplementary Information). Additionally, a few studies reported the thickness of lamellar (not the thickness of each layer) that were consistent across fiber bundles and species (11 nm in rat spinal cord (Chen et al., 2017; Rosenbluth et al., 1996), $10.7 \mathrm{~nm}$ in rat optic nerve and white matter (Karlsson, 1966), $11.2 \mathrm{~nm}$ in dog spinal cord (Raine, 1984)). These results further support that the robustness of the scaling parameter. Recently, a study demonstrated that thickness of lamellar does not change during a demyelinating disease as long as the lamellar structure is intact (Chen et al., 2017), suggesting the potential applicability of the scaling parameter in pathological conditions. However, one has to be cautious in applying the method for pathological conditions (see "accuracy of g-ratio mapping” in Discussion).

The other parameter for the scaling was $\kappa_{a x}\left(=\kappa_{e x}\right)$, which was calculated as 0.86 in the mass-density approach. In other studies, the parameter was suggested to be 0.8 (Finean, 1960), 0.82 (Marjo and van der Knaap, 2005), and 0.9 (LoPachin et al., 1991). When a computer simulation was performed, MVF and g-ratio showed little change for the range of variations $(0.8$ to 0.9) in $\kappa_{a x}$, supporting the robustness of our scaling approach (see Supplementary Information). Note that all the parameters reported in this study are from the measurements of mammalian CNS.

In addition to the histological validation results in our study, the application of the same scaling factors can be demonstrated using another study that measured both MWF and MVF (Dula et al., 2010). In the study, MVF of rat spinal cords using light microscopy was reported to range from $51 \%$ to $61 \%$ (these values are larger than that reported in other studies (Benninger et al., 2006; 
Chomiak and $\mathrm{Hu}, 2009$ )), and MWF using MRI was measured to range from 29\% to $36 \%$ (except dorsal CST, which has underestimated MWF due to water exchange). When MWF was translated into MVF using our scaling approach, MVF was estimated to range from $49 \%$ to $57 \%$. This result demonstrates the consistency of the proposed scaling approach for different species (rat vs. mouse), different regions (spinal cord vs. brain), and different field strengths (7T vs. 15.2T) in the two studies (Dula et al., 2010; West et al., 2016b). Compared to these studies, which scanned rodents using spin echo MWI (SE-MWI), we applied the method for human using GRE-MWI. Because of the aforementioned consistency in the scaling parameters across regions and species, we believe they still hold for the human brain. The two MWI methods (SE-MWI and GRE-MWI) have recently demonstrated to provide similar MWF measurements (Alonso-Ortiz et al., 2017).

\section{Geometric approach vs. mass-density approach}

When comparing the $\kappa_{m y}$ value from mass-density approach with that from the geometric approach, they showed similar values ( 0.39 in the mass-density approach vs. 0.36 in geometric approach). The difference may originate from the water molecules in the lipid bilayer in the mass-density approach. It was reported that water molecules exist in the myelin lipid bilayer and account for $12 \%$ of total water molecules in myelin sheath (Vandenheuvel, 1965). These water molecules may not be visible in MRI due to extremely short $\mathrm{T}_{2}$ and, therefore, need to be excluded in estimating $\kappa_{m y}$. In the mass-density approach, however, all water molecules including those in lipid layers are incorrectly classified as the MR visible volume because the water mass fraction of myelin is calculated by comparing the weight of wet and dry white matter (Laule et al., 2004; Marjo and van der Knaap, 2005). Hence, $\kappa_{m y}$ is overestimated in the mass-density approach. On the other hand, the geometric approach does not take into account the water molecules in the lipid layer.

In the geometric approach, other molecules such as proteins in the myelin water layer are ignored, assuming the concentration is small. This assumption is reasonable since the concentration has been reported to be less than 5\% (Gennis, 1989; West et al., 2016b).

Recently, a study estimated $\kappa_{m y}$ using the mass-density approach (West et al., 2016b). 
The resulting $\kappa_{m y}$ value $(=0.475)$ was higher than ours $(=0.39$ in the mass-density approach). The difference may originate from the difference in the water content of myelin (Laule et al., 2004; West et al., 2016b), which is defined as the mass of water molecules in myelin per $1 \mathrm{~g}$ of myelin. Note that the parameters used in the mass-density approach have been shown to vary with tissue fixation, leading to an error in the measurements (Chen et al., 2017; Finean, 1960). On the other hand, the thicknesses of the lamellar structure used in the geometric approach were measured in fresh tissues (Finean, 1962; Kirschner and Caspar, 1972) and were not affected by fixation. Thus, $\kappa_{m y}$ estimated in the geometric approach may provide a more accurate result.

\section{Estimation of MVF using MRI}

In MRI, a few methods including MWI and MT have been suggested to be sensitive to myelin concentration. Compared to MT-based methods, our methods of scaling MWI to MVF utilize geometric parameters (e.g. thickness of myelin water layer and lipid layer) and have demonstrated consistent scaling parameters for both geometric and mass-density approaches $(0.36$ and 0.39 respectively). On the other hand, MT-based methods have shown widely varying scaling factors (1.6 to 5.2) (Dula et al., 2010; Stikov et al., 2015; Thiessen et al., 2013). In terms of data acquisition, proposed GRE-based MWI approach is efficient, providing a whole brain myelin map in less than 10 mins. Further reduction of the scan time by optimizing the scan parameters and by using advanced methods (e.g. multi-band imaging) can be achieved, making the method potentially more appealing than MT-based methods.

In our study, the mean g-ratio value of all ROI is $0.78 \pm 0.05$ (from 0.71 to 0.85 ) and is close to the theoretically optimum g-ratio of 0.77 (Chomiak and $\mathrm{Hu}, 2009$ ). This range of g-ratio is in good agreement with most of histological g-ratio studies listed in Table 1 (Arnett et al., 2001; Benninger et al., 2006; Blakemore, 1974; Chau et al., 2000; Chomiak and Hu, 2009; Graf von Keyserlingk and Schramm, 1984; Guy et al., 1989; Liewald et al., 2014; Mason et al., 2001; Waxman and Swadlow, 1976; West et al., 2016a). The result, however, is slightly higher than those from the previous studies using MT approaches for MVF estimation (Table 1) (Cercignani et al., 2017; Mohammadi et al., 2015; Stikov et al., 2015). Several factors may account for the difference. For example, scaling of an MT parameter with a zero intercept has been used in a few studies, 
which may suffer from the nonzero intercept demonstrated in more recent studies (Campbell et al., 2017; West et al., 2016b). Additionally, the approach of scaling the g-ratio of the splenium to 0.7, which has been used in a few previous studies, may have resulted in a bias in the g-ratio map. Note that the assumption of the splenium to have a g-ratio of 0.7 is not supported histologically because the reference (Graf von Keyserlingk and Schramm, 1984) does not clearly state a tract-specific gratio.

Recently, new approaches such as macromolecular tissue volume (MTV) imaging (Mezer et al., 2013), ultrashort echo time (UTE) myelin proton imaging (Du et al., 2014; Wilhelm et al., 2012), and direct visualization of short transverse relaxation time component (ViSTa) imaging (Oh et al., 2013) have been proposed to acquire myelin sensitive contrasts. These methods also require certain assumptions or scaling factors that translate myelin concentration measurements to myelin volume fraction. For example, the studies that applied MTV for g-ratio mapping suggested to use unscaled MTV values, assuming the volume of non-myelin macromolecules (e.g. glial cells) is equal to myelin water volume (Berman et al., 2017; Duval et al., 2017).

\section{Accuracy of g-ratio mapping}

Despite our efforts of accurately estimating each parameter, there are a few potential complications that can bias our results. First, MVF can be underestimated by inter-compartmental water exchange between myelin water and axonal and extracellular water (Dula et al., 2010; Kalantari et al., 2011; Sled and Pike, 2001). Secondly, GRE-MWI is sensitive to field inhomogeneity, generating image artifacts at the frontal lobe. The artifacts may be the source for the large inter-subject COV of MVF in GN (9.0\%). Recently, methods have been proposed to mitigate the artifacts (Alonso-Ortiz et al., 2016; Lee et al., 2017). In AVF estimated using NODDI, a few parameters (e.g. parallel diffusivity of the axon and extracellular compartment) are fixed in the model, which may lead to estimation errors in AVF (Jelescu et al., 2016; Novikov et al., 2015). Another limitation of our method is that it may not properly estimate g-ratio in gray matter because of the low concentration and low CNR of MVF (Meyers et al., 2009) and the inaccurate estimation of AVF (Guerrero et al., 2016; Lampinen et al., 2017). Lastly, MWF estimation may not distinguish between intact myelin and myelin debris with intact lamellar structure (Chen et al., 2017). This 
may result in an unwanted overestimation of MVF in the early stage of demyelination. Furthermore, one has to be extra careful in estimating and interpreting the parametric results in pathological conditions since the models may not correctly explain the conditions (e.g. myelin edema) (Harkins et al., 2013).

\section{CONCLUSION}

In this study, we demonstrated an approach of acquiring the whole brain in-vivo g-ratio map from the human brain. The approach combined GRE-MWI and NODDI to estimate MVF and AVF, respectively, and provided a reliable estimation of the g-ratio map. In generating the g-ratio map, we developed a new scaling method to translate MWF of GRE-MWI into MVF using the geometric measures of myelin structure. The proposed g-ratio mapping approach may assist us to explore microstructural differences and changes between fiber bundles and different groups.

\section{ACKNOWLEDGEMENT}

We sincerely thank Drs. Mark D. Does and Kathyrn L. West for sharing histo-imaging data (supported by NIH R01-EB019980). This research was supported by the Brain Research Program through the National Research Foundation of Korea (NRF) funded by the Ministry of Science, ICT \& Future Planning (NRF-2015M3C7A1031969). 


\section{REFERENCES}

Albert, M., Antel, J., Brück, W., Stadelmann, C., 2007. Extensive cortical remyelination in patients with chronic multiple sclerosis. Brain pathology 17, 129-138.

Alonso-Ortiz, E., Levesque, I.R., Pike, G.B., 2017. Multi-gradient-echo myelin water fraction imaging: Comparison to the multi-echo-spin-echo technique. Magn Reson Med.

Alonso-Ortiz, E., Levesque, I.R., Paquin, R., Pike, G.B., 2016. Field inhomogeneity correction for gradient echo myelin water fraction imaging. Magn Reson Med.

Andersson, J.L., Skare, S., Ashburner, J., 2003. How to correct susceptibility distortions in spinecho echo-planar images: application to diffusion tensor imaging. Neuroimage 20, 870-888.

Arnett, H.A., Mason, J., Marino, M., Suzuki, K., Matsushima, G.K., Ting, J.P.-Y., 2001. TNF $\alpha$ promotes proliferation of oligodendrocyte progenitors and remyelination. Nature neuroscience 4 , 1116-1122.

Avants, B.B., Tustison, N., Song, G., 2009. Advanced normalization tools (ANTS). Insight J 2, 135.

Benninger, Y., Colognato, H., Thurnherr, T., Franklin, R.J., Leone, D.P., Atanasoski, S., Nave, K.A., Suter, U., Relvas, J.B., 2006. $\beta 1$-integrin signaling mediates premyelinating oligodendrocyte survival but is not required for CNS myelination and remyelination. The Journal of Neuroscience 26, 7665-7673.

Berman, S., West, K.L., Does, M.D., Yeatman, J.D., Mezer, A.A., 2017. Evaluating g-ratio weighted changes in the corpus callosum as a function of age and sex. Neuroimage.

Blakemore, W., 1974. Pattern of remyelination in the CNS.

Bouyagoub, S., Dowell, N.G., Hurely, S.A., Wood, T.C., Cercignani, M., 2016. Overestimation of CSF fraction in NODDI: possible correction techniques and the effect on neurite density and orientation dispersion measures. Proceeding of the 24th Annual Meeting of the ISMRM, Singapore, p. 0007.

Buonocore, M.H., Gao, L., 1997. Ghost artifact reduction for echo planar imaging using image phase correction. Magn Reson Med 38, 89-100.

Campbell, J.S., Leppert, I.R., Narayanan, S., Duval, T., Cohen-Adad, J., Pike, G.B., Stikov, N., 2017. Promise and pitfalls of g-ratio estimation with MRI. arXiv preprint arXiv:1701.02760.

Cercignani, M., Giulietti, G., Dowell, N.G., Gabel, M., Broad, R., Leigh, P.N., Harrison, N.A., 
Bozzali, M., 2017. Characterizing axonal myelination within the healthy population: a tract-bytract mapping of effects of age and gender on the fiber g-ratio. Neurobiology of Aging 49, 109118.

Chau, W.K., So, K.-F., Tay, D., Dockery, P., 2000. A morphometric study of optic axons regenerated in a sciatic nerve graft of adult rats. Restorative neurology and neuroscience 16, 105 116.

Chen, H.S.-M., Holmes, N., Liu, J., Tetzlaff, W., Kozlowski, P., 2017. Validating Myelin Water Imaging with Transmission Electron Microscopy in a Rat Spinal Cord Injury Model. Neuroimage $153,122-130$.

Chomiak, T., Hu, B., 2009. What is the optimal value of the g-ratio for myelinated fibers in the rat CNS? A theoretical approach. PLoS One 4, e7754.

Daducci, A., Canales-Rodríguez, E.J., Zhang, H., Dyrby, T.B., Alexander, D.C., Thiran, J.-P., 2015. Accelerated microstructure imaging via convex optimization (AMICO) from diffusion MRI data. Neuroimage 105, 32-44.

De Santis, S., Assaf, Y., Jeurissen, B., Jones, D.K., Roebroeck, A., 2016. T1 relaxometry of crossing fibres in the human brain. Neuroimage 141, 133-142.

Dean, D.C., O'Muircheartaigh, J., Dirks, H., Travers, B.G., Adluru, N., Alexander, A.L., Deoni, S.C., 2016. Mapping an index of the myelin g-ratio in infants using magnetic resonance imaging. Neuroimage 132, 225-237.

Deoni, S.C., Rutt, B.K., Arun, T., Pierpaoli, C., Jones, D.K., 2008. Gleaning multicomponent $T_{1}$ and $\mathrm{T}_{2}$ information from steady-state imaging data. Magn Reson Med 60, 1372-1387.

Du, J., Ma, G., Li, S., Carl, M., Szeverenyi, N.M., VandenBerg, S., Corey-Bloom, J., Bydder, G.M., 2014. Ultrashort echo time (UTE) magnetic resonance imaging of the short $\mathrm{T}_{2}$ components in white matter of the brain using a clinical 3T scanner. Neuroimage 87, 32-41.

Du, Y.P., Chu, R., Hwang, D., Brown, M.S., Kleinschmidt-DeMasters, B.K., Singel, D., Simon, J.H., 2007. Fast multislice mapping of the myelin water fraction using multicompartment analysis of $\mathrm{T}_{2} *$ decay at 3T: a preliminary postmortem study. Magn Reson Med 58, 865-870.

Dula, A.N., Gochberg, D.F., Valentine, H.L., Valentine, W.M., Does, M.D., 2010. Multiexponential $\mathrm{T}_{2}$, magnetization transfer, and quantitative histology in white matter tracts of rat spinal cord. Magn Reson Med 63, 902-909.

Duval, T., Lévy, S., Stikov, N., Campbell, J., Mezer, A., Witzel, T., Keil, B., Smith, V., Wald, L.L., 
Klawiter, E., 2017. g-Ratio weighted imaging of the human spinal cord in vivo. Neuroimage 145, 11-23.

Finean, J., 1962. The nature and stability of the plasma membrane. Circulation 26, 1151-1162.

Finean, J.B., 1960. Electron microscope and x-ray diffraction studies of the effects of dehydrations on the structure of nerve myelin. I. Peripheral nerve. J Biophys Biochem Cytol 8, 13-29.

Gennis, R.B., 1989. Biomembranes: Molecular Structure and Function. Springer-Verlag, New York.

Goldman, L., Albus, J.S., 1968. Computation of impulse conduction in myelinated fibers; theoretical basis of the velocity-diameter relation. Biophysical journal 8, 596-607.

Graf von Keyserlingk, D., Schramm, U., 1984. Diameter of axons and thickness of myelin sheaths of the pyramidal tract fibres in the adult human medullary pyramid. Anat Anz 157, 97-111.

Guerrero, J.M., Adluru, N., Kecskemeti, S.R., Davidson, R.J., Alexander, A.L., 2016. Investigating the effects of intrinsic diffusivity on neurite orientation dispersion and density imaging (NODDI). Proceeding of the 24th Annual Meeting of the ISMRM, Singapore, p. 1046.

Guy, J., Ellis, E., Kelley, K., Hope, G.M., 1989. Spectra of G ratio, myelin sheath thickness, and axon and fiber diameter in the guinea pig optic nerve. Journal of Comparative Neurology 287, 446-454.

Hammond, K.E., Lupo, J.M., Xu, D., Metcalf, M., Kelley, D.A., Pelletier, D., Chang, S.M., Mukherjee, P., Vigneron, D.B., Nelson, S.J., 2008. Development of a robust method for generating 7.0 T multichannel phase images of the brain with application to normal volunteers and patients with neurological diseases. Neuroimage 39, 1682-1692.

Harkins, K.D., Valentine, W.M., Gochberg, D.F., Does, M.D., 2013. In-vivo multi-exponential T2, magnetization transfer and quantitative histology in a rat model of intramyelinic edema. NeuroImage: Clinical 2, 810-817.

Jelescu, I.O., Veraart, J., Fieremans, E., Novikov, D.S., 2016. Degeneracy in model parameter estimation for multi-compartmental diffusion in neuronal tissue. NMR Biomed 29, 33-47.

Jenkinson, M., Beckmann, C.F., Behrens, T.E., Woolrich, M.W., Smith, S.M., 2012. Fsl. Neuroimage 62, 782-790.

Jenkinson, M., Pechaud, M., Smith, S., 2005. BET2: MR-based estimation of brain, skull and scalp surfaces. Eleventh annual meeting of the organization for human brain mapping. Toronto, ON, p. 167. 
Kalantari, S., Laule, C., Bjarnason, T.A., Vavasour, I.M., MacKay, A.L., 2011. Insight into in vivo magnetization exchange in human white matter regions. Magn Reson Med 66, 1142-1151.

Karlsson, U., 1966. Comparison of the myelin period of peripheral and central origin by electron microscopy. Journal of ultrastructure research 15, 451-468.

Kirschner, D., Caspar, D., 1972. Comparative diffraction studies on myelin membranes. Ann N Y Acad Sci 195, 309-320.

Kitajima, M., Korogi, Y., Takahashi, M., Eto, K., 1996. MR signal intensity of the optic radiation. AJNR Am J Neuroradiol 17, 1379-1383.

Lampinen, B., Szczepankiewicz, F., Martensson, J., van Westen, D., Sundgren, P.C., Nilsson, M., 2017. Neurite density imaging versus imaging of microscopic anisotropy in diffusion MRI: A model comparison using spherical tensor encoding. Neuroimage 147, 517-531.

Lancaster, J.L., Andrews, T., Hardies, L.J., Dodd, S., Fox, P.T., 2003. Three-pool model of white matter. J Magn Reson Imaging 17, 1-10.

Laule, C., Kozlowski, P., Leung, E., Li, D.K., MacKay, A.L., Moore, G.W., 2008. Myelin water imaging of multiple sclerosis at 7 T: correlations with histopathology. Neuroimage 40, 1575-1580. Laule, C., Leung, E., Li, D.K., Traboulsee, A., Paty, D., MacKay, A., Moore, G.R., 2006. Myelin water imaging in multiple sclerosis: quantitative correlations with histopathology. Multiple Sclerosis Journal 12, 747-753.

Laule, C., Vavasour, I., Moore, G., Oger, J., Li, D., Paty, D., MacKay, A., 2004. Water content and myelin water fraction in multiple sclerosis. Journal of neurology 251, 284-293.

Lee, D., Lee, J., Lee, J., Nam, Y., 2017. Single-Scan GRE Myelin Water Imaging with Macroscopic Field Inhomogeneity Compensation. Proceeding of the 25th Annual Meeting of the ISMRM, Honolulu, HI, USA, p. 1399.

Liewald, D., Miller, R., Logothetis, N., Wagner, H.-J., Schüz, A., 2014. Distribution of axon diameters in cortical white matter: an electron-microscopic study on three human brains and a macaque. Biological cybernetics 108, 541-557.

LoPachin, R.M., Castiglia, C.M., Saubermann, A.J., 1991. Elemental composition and water content of myelinated axons and glial cells in rat central nervous system. Brain research 549, 253259.

Lu, W., Yu, H., Shimakawa, A., Alley, M., Reeder, S.B., Hargreaves, B.A., 2008. Water-fat separation with bipolar multiecho sequences. Magn Reson Med 60, 198-209. 
Mackay, A., Whittall, K., Adler, J., Li, D., Paty, D., Graeb, D., 1994. In vivo visualization of myelin water in brain by magnetic resonance. Magn Reson Med 31, 673-677.

Marjo, S., van der Knaap, J., 2005. Magnetic resonance of myelination and myelin disorders. Magnetic resonance of myelination and myelin disorders.

Mason, J., Langaman, C., Morell, P., Suzuki, K., Matsushima, G.K., 2001. Episodic demyelination and subsequent remyelination within the murine central nervous system: changes in axonal calibre. Neuropathology and applied neurobiology 27, 50-58.

Meyers, S.M., Laule, C., Vavasour, I.M., Kolind, S.H., Mädler, B., Tam, R., Traboulsee, A.L., Lee, J., Li, D.K., MacKay, A.L., 2009. Reproducibility of myelin water fraction analysis: a comparison of region of interest and voxel-based analysis methods. Magnetic resonance imaging 27, 10961103.

Mezer, A., Yeatman, J.D., Stikov, N., Kay, K.N., Cho, N.-J., Dougherty, R.F., Perry, M.L., Parvizi, J., Hua, L.H., Butts-Pauly, K., 2013. Quantifying the local tissue volume and composition in individual brains with magnetic resonance imaging. Nature medicine 19, 1667-1672.

Mierzwa, A., Shroff, S., Rosenbluth, J., 2010. Permeability of the paranodal junction of myelinated nerve fibers. The Journal of Neuroscience 30, 15962-15968.

Mohammadi, S., Carey, D., Dick, F., Diedrichsen, J., Sereno, M.I., Reisert, M., Callaghan, M.F., Weiskopf, N., 2015. Whole-brain in-vivo measurements of the axonal g-ratio in a group of 37 healthy volunteers. Front Neurosci 9, 441.

Morell, P., Quarles, R.H., 1999. Myelin formation, structure and biochemistry.

Nam, Y., Kim, D.-H., Lee, J., 2015a. Physiological noise compensation in gradient-echo myelin water imaging. Neuroimage 120, 345-349.

Nam, Y., Lee, J., Hwang, D., Kim, D.H., 2015b. Improved estimation of myelin water fraction using complex model fitting. Neuroimage 116, 214-221.

Novikov, D.S., Jelescu, I.O., Fieremans, E., 2015. From diffusion signal moments to neurite diffusivities, volume fraction and orientation distribution: an exact solution. Proceeding of the 23rd Annual Meeting of the ISMRM, Canada, p. 469.

Odrobina, E.E., Lam, T.Y., Pun, T., Midha, R., Stanisz, G.J., 2005. MR properties of excised neural tissue following experimentally induced demyelination. NMR Biomed 18, 277-284.

Oh, S.-H., Bilello, M., Schindler, M., Markowitz, C.E., Detre, J.A., Lee, J., 2013. Direct visualization of short transverse relaxation time component (ViSTa). Neuroimage 83, 485-492. 
Peters, A., 1960. The formation and structure of myelin sheaths in the central nervous system. J Biophys Biochem Cytol 8, 431-446.

Raine, C.S., 1984. Morphology of myelin and myelination. Myelin. Springer, pp. 1-50.

Rosenbluth, J., Liang, W.-L., Liu, Z., Guo, D., Schiff, R., 1996. Expanded CNS myelin sheaths formed in situ in the presence of an IgM antigalactocerebroside-producing hybridoma. Journal of Neuroscience 16, 2635-2641.

Rushton, W.A., 1951. A theory of the effects of fibre size in medullated nerve. J Physiol 115, 101122.

Schmierer, K., Tozer, D.J., Scaravilli, F., Altmann, D.R., Barker, G.J., Tofts, P.S., Miller, D.H., 2007. Quantitative magnetization transfer imaging in postmortem multiple sclerosis brain. Journal of magnetic resonance imaging 26, 41-51.

Sled, J.G., Pike, G.B., 2001. Quantitative imaging of magnetization transfer exchange and relaxation properties in vivo using MRI. Magn Reson Med 46, 923-931.

Stikov, N., Campbell, J.S., Stroh, T., Lavelee, M., Frey, S., Novek, J., Nuara, S., Ho, M.K., Bedell, B.J., Dougherty, R.F., Leppert, I.R., Boudreau, M., Narayanan, S., Duval, T., Cohen-Adad, J., Picard, P.A., Gasecka, A., Cote, D., Pike, G.B., 2015. In vivo histology of the myelin g-ratio with magnetic resonance imaging. Neuroimage 118, 397-405.

Stikov, N., Perry, L.M., Mezer, A., Rykhlevskaia, E., Wandell, B.A., Pauly, J.M., Dougherty, R.F., 2011. Bound pool fractions complement diffusion measures to describe white matter micro and macrostructure. Neuroimage 54, 1112-1121.

Thiessen, J.D., Zhang, Y., Zhang, H., Wang, L., Buist, R., Del Bigio, M.R., Kong, J., Li, X.M., Martin, M., 2013. Quantitative MRI and ultrastructural examination of the cuprizone mouse model of demyelination. NMR Biomed 26, 1562-1581.

Vandenheuvel, F.A., 1965. Structural studies of biological membranes: the structure of myelin. Ann N Y Acad Sci 122, 57-76.

Waxman, S.G., 1980. Determinants of conduction velocity in myelinated nerve fibers. Muscle \& nerve $3,141-150$.

Waxman, S.G., Swadlow, H.A., 1976. Ultrastructure of visual callosal axons in the rabbit. Experimental neurology 53, 115-127.

West, K.L., Kelm, N.D., Carson, R.P., Does, M.D., 2016a. A revised model for estimating g-ratio from MRI. Neuroimage 125, 1155-1158. 
West, K.L., Kelm, N.D., Carson, R.P., Gochberg, D.F., Ess, K.C., Does, M.D., 2016b. Myelin volume fraction imaging with MRI. Neuroimage.

Wilhelm, M.J., Ong, H.H., Wehrli, S.L., Li, C., Tsai, P.-H., Hackney, D.B., Wehrli, F.W., 2012. Direct magnetic resonance detection of myelin and prospects for quantitative imaging of myelin density. Proceedings of the National Academy of Sciences 109, 9605-9610.

Wolff, S.D., Balaban, R.S., 1989. Magnetization transfer contrast (MTC) and tissue water proton relaxation in vivo. Magn Reson Med 10, 135-144.

Yagishita, A., Nakano, I., Oda, M., Hirano, A., 1994. Location of the corticospinal tract in the internal capsule at MR imaging. Radiology 191, 455-460.

Zhang, H., Schneider, T., Wheeler-Kingshott, C.A., Alexander, D.C., 2012. NODDI: practical in vivo neurite orientation dispersion and density imaging of the human brain. Neuroimage 61, 10001016. 